\title{
WEIGHTED ESTIMATES FOR COMMUTATORS ON NONHOMOGENEOUS SPACES
}

\author{
WENGU CHEN AND BING ZHAO
}

Received 14 November 2005; Revised 8 March 2006; Accepted 11 March 2006

Let $\mu$ be a Borel measure on $\mathbb{R}^{d}$ which may be nondoubling. The only condition that $\mu$ must satisfy is $\mu(Q) \leq c_{0} l(Q)^{n}$ for any cube $Q \subset \mathbb{R}^{d}$ with sides parallel to the coordinate axes and for some fixed $n$ with $0<n \leq d$. This paper is to establish the weighted norm inequality for commutators of Calderón-Zygmund operators with $\operatorname{RBMO}(\mu)$ functions by an estimate for a variant of the sharp maximal function in the context of the nonhomogeneous spaces.

Copyright (c) 2006 W. Chen and B. Zhao. This is an open access article distributed under the Creative Commons Attribution License, which permits unrestricted use, distribution, and reproduction in any medium, provided the original work is properly cited.

\section{Introduction}

Let $\mu$ be some nonnegative Borel measure on $\mathbb{R}^{d}$ satisfying

$$
\mu(Q) \leq c_{0} l(Q)^{n}
$$

for any cube $Q \subset \mathbb{R}^{d}$ with sides parallel to the coordinate axes, where $l(Q)$ stands for the side length of $Q$ and $n$ is a fixed real number such that $0<n \leq d$. Throughout this paper, all cubes we will consider will be those with sides parallel to the coordinate axes. For $r>0, r Q$ will denote the cube with the same center as $Q$ and with $l(r Q)=r l(Q)$. Moreover, $Q(x, r)$ will be the cube centered at $x$ with side length $r$.

The classical theory of harmonic analysis for maximal functions and singular integrals on $\left(\mathbb{R}^{d}, \mu\right)$ has been developed under the assumption that the underlying measure $\mu$ satisfies the doubling property, that is, there exists a constant $c>0$ such that $\mu(B(x, 2 r)) \leq c \mu(B(x, r))$ for every $x \in \mathbb{R}^{d}$ and $r>0$. But recently, many classical results have been proved still valid without the doubling condition; see [1-18] and their references.

Orobitg and Pérez [11] have studied an analogue of the classical theory of $A_{p}(\mu)$ weights in $\mathbb{R}^{d}$ without assuming that the underlying measure $\mu$ is doubling. Then, they 
obtained weighted norm inequalities for the (centered) Hardy-Littlewood maximal function and corresponding weighted estimates for nonclassical Calderón-Zygmund operators. They also considered commutators of those Calderón-Zygmund operators with $\operatorname{BMO}(\mu)$ functions. The purpose of this paper is to establish weighted estimates for commutators of those nonclassical Calderón-Zygmund operators with $\operatorname{RBMO}(\mu)$ in this new setting.

Let us introduce some notations and definitions. Given two cubes $Q \subset R$ in $\mathbb{R}^{d}$, we set

$$
K_{Q, R}=1+\sum_{k=1}^{N_{Q, R}} \frac{\mu\left(2^{k} Q\right)}{l\left(2^{k} Q\right)^{n}}
$$

where $N_{Q, R}$ is the first integer $k$ such that $l\left(2^{k} Q\right) \geq l(R) . K_{Q, R}$ was introduced by Tolsa in [15].

Given $\beta_{d}$ (depending on $d$ ) big enough (e.g., $\beta_{d}>2^{n}$ ), we say that some cube $Q \subset \mathbb{R}^{d}$ is doubling if $\mu(2 Q) \leq \beta_{d} \mu(Q)$.

Given a cube $Q \subset \mathbb{R}^{d}$, let $N$ be the smallest integer $\geq 0$ such that $2^{N} Q$ is doubling. We denote this cube by $\widetilde{Q}$.

Let $\eta>1$ be some fixed constant. We say that a function $b(x)$ is in $\operatorname{RBMO}(\mu)$ if there exists some constant $c_{1}$ such that for any cube $Q$,

$$
\begin{gathered}
\frac{1}{\mu(\eta Q)} \int_{Q}\left|b-m_{\widetilde{Q}} b\right| d \mu \leq c_{1}, \\
\left|m_{Q} b-m_{R} b\right| \leq c_{1} K_{Q, R} \quad \text { for any two doubling cubes } Q \subset R,
\end{gathered}
$$

where $m_{Q} b=1 / \mu(Q) \int_{Q} b d \mu$. The minimal constant $c_{1}$ is the $\operatorname{RBMO}(\mu)$ norm of $b$, and it will be denoted by $\|b\|_{*}$. The $\operatorname{RBMO}(\mu)$ function space was introduced by Tolsa in [15] and shares more properties with the classical $\mathrm{BMO}$ function space than $\mathrm{BMO}(\mu)$ space.

We say a kernel $k(x, y): \mathbb{R}^{d} \times \mathbb{R}^{d} \backslash\{(x, y): x=y\} \rightarrow \mathbb{C}$ is an $n$-dimensional CalderónZygmund kernel in the new setting if

(1) $|k(x, y)| \leq A /|x-y|^{n}$ if $x \neq y$,

(2) there exists $0<\gamma \leq 1$ such that

$$
\left|k(x, y)-k\left(x^{\prime}, y\right)\right|+\left|k(y, x)-k\left(y, x^{\prime}\right)\right| \leq \frac{A\left|x-x^{\prime}\right|^{\gamma}}{|x-y|^{n+\gamma}}
$$

if $|x-y|>2\left|x-x^{\prime}\right|$.

A bounded linear operator $T$ from $L^{2}(\mu)$ to $L^{2}(\mu)$ is said to be a Calderón-Zygmund operator with $n$-dimensional kernel $k$ if for every compacted supported function $f \in$ $L^{2}(\mu)$

$$
T f(x)=\int_{\mathbb{R}^{d}} k(x, y) f(y) d \mu(y) \quad \text { for } x \notin \operatorname{supp} f .
$$

For $r>0$, we define the truncated operators by

$$
T_{r} f(x)=\int_{\mathbb{R}^{d} \backslash B(x, r)} k(x, y) f(y) d \mu(y)
$$


and define the maximal operator associated with $T$ as follows:

$$
T_{*} f(x)=\sup _{r>0}\left|T_{r} f(x)\right|
$$

\section{Sharp maximal function estimates for commutators}

In [15], Tolsa defined a sharp maximal operator $M^{\#} f(x)$ such that

$$
f \in \operatorname{RBMO}(\mu) \Longleftrightarrow M^{\#} f \in L^{\infty}(\mu),
$$

where

$$
M^{\#} f(x)=\sup _{x \in Q} \frac{1}{\mu((3 / 2) Q)} \int_{Q}\left|f-m_{\widetilde{Q}} f\right| d \mu+\sup _{\substack{x \in Q \subset R \\ Q, R \text { doubling }}} \frac{\left|m_{Q} f-m_{R} f\right|}{K_{Q, R}} .
$$

We also consider the noncentered doubling maximal operator $N$ :

$$
N f(x)=\sup _{\substack{x \in Q \\ Q \text { doubling }}} \frac{1}{\mu(Q)} \int_{Q}|f| d \mu .
$$

By [15, Remark 2.3], for $\mu$-almost all $x \in \mathbb{R}^{d}$ one can find a sequence of doubling cubes $\left\{Q_{k}\right\}_{k}$ centered at $x$ with $l\left(Q_{k}\right) \rightarrow 0$ as $k \rightarrow \infty$ such that

$$
\lim _{k \rightarrow \infty} \frac{1}{\mu\left(Q_{k}\right)} \int_{Q_{k}} b(y) d \mu(y)=b(x) .
$$

So, $|f(x)| \leq N f(x)$ for $\mu$-a.e. $x \in \mathbb{R}^{d}$. Moreover, it is easy to show that $N$ is of weak type $(1,1)$ and bounded on $L^{p}(\mu), p \in(1, \infty]$.

In order to obtain the estimate for a variant of the sharp maximal function for the commutators of Calderón-Zygmund operators defined as above with $\operatorname{RBMO}(\mu)$ functions, we need the following definition.

A function $B:[0, \infty) \rightarrow[0, \infty)$ is called a Young function if it is continuous, convex, increasing, and satisfying $B(0)=0$ and $B(t) \rightarrow \infty$ as $t \rightarrow \infty$. We define the $B$-average of a function $f$ over a cube $Q$ by means of the following Luxemburg norm:

$$
\|f\|_{B, Q,(\rho)}=\inf \left\{\lambda>0: \frac{1}{\mu(\rho Q)} \int_{Q} B\left(\frac{|f(y)|}{\lambda}\right) d \mu \leq 1\right\} .
$$

The generalized Hölder's inequality

$$
\frac{1}{\mu(\rho Q)} \int_{Q}|f(y) g(y)| d \mu(y) \leq\|f\|_{B, Q,(\rho)}\|g\|_{\bar{B}, Q,(\rho)}
$$

holds, where $\bar{B}$ is the complementary Young function associated to $B$. For every locally integrable function $f$, define its maximal operator $M_{B,(\rho)}$ by

$$
M_{B,(\rho)} f(x)=\sup _{x \in Q}\|f\|_{B, Q,(\rho)} .
$$


Theorem 2.1. Let $b \in \operatorname{RBMO}(\mu)$, let $0<\delta<\epsilon<1$, there exists $C=C_{\delta, \epsilon}$ such that

$$
M_{\delta}^{\#}([b, T] f)(x) \leq C\|b\|_{*}\left(M_{\epsilon,(3 / 2)}(T f)(x)+M_{(9 / 8)}^{2} f(x)+T_{*} f(x)\right)
$$

where $M_{\delta}^{\#} f(x)=M^{\#}\left(|f|^{\delta}\right)^{1 / \delta}, M_{p,(\rho)} f(x)=\sup _{x \in Q}\left((1 / \mu(\rho Q)) \int_{Q}|f|^{p} d \mu\right)^{1 / p}, 0<p<\infty$. Set $M_{(\rho)} f(x)=M_{1,(\rho)} f(x)$.

Before proving the theorem, another equivalent norm for $\operatorname{RBMO}(\mu)$ is needed. Suppose that for a given function $b \in L_{\text {loc }}^{1}(\mu)$ there exist some $c_{2}$ and a collection of numbers $\left\{b_{Q}\right\}_{Q}$ (i.e., for each cube $Q$, there exists $b_{Q} \in \mathbb{R}$ ) such that

$$
\begin{gathered}
\sup _{Q} \frac{1}{\mu(\eta Q)} \int_{Q}\left|b-b_{Q}\right| d \mu \leq c_{2}, \\
\left|b_{Q}-b_{R}\right| \leq c_{2} K_{Q, R} \quad \text { for any two cubes } Q \subset R .
\end{gathered}
$$

Then, set $\|b\|_{* *}=\inf c_{2}$, where the infimum is taken over all the constants $c_{2}$ and all the numbers $\left\{b_{Q}\right\}$ satisfying (2.9). By [15, Lemma 2.8, page 99], for a fixed $\eta>1$, the norms $\|\cdot\|_{*}$ and $\|\cdot\|_{* *}$ are equivalent.

Proof of Theorem 2.1. We follow the argument from [15, proof of Theorem 9.1]. Let $Q=$ $Q(x, r)$ be a cube with center $x$ and side length $r$. For $0<\delta<1$ and $\alpha, \beta \in \mathbb{R}$, we have $\left.|| \alpha\right|^{\delta}-|\beta|^{\delta}|\leq| \alpha-\left.\beta\right|^{\delta}$. Let $\left\{b_{Q}\right\}_{Q}$ be a sequence of numbers satisfying

$$
\int_{Q}\left|b-b_{Q}\right| d \mu \leq 2 \mu(2 Q)\|b\|_{* *}
$$

for all cubes $Q$ and

$$
\left|b_{Q}-b_{R}\right| \leq 2 K_{Q, R}\|b\|_{* *}
$$

for all cubes $Q, R$ with $Q \subset R$. For any cube $Q$, we denote $h_{Q}:=-m_{Q}\left(T\left(\left(b-b_{Q}\right) f \chi_{\mathbb{R}^{d}} \backslash\right.\right.$ $(4 / 3) Q))$. We will show that for all $x, Q$ with $x \in Q$,

$$
\frac{1}{\mu((3 / 2) Q)}\left(\int_{Q}\left|[b, T] f-h_{Q}\right|^{\delta} d \mu\right)^{1 / \delta} \leq C\|b\|_{* *}\left(M_{\epsilon,(3 / 2)}(T f)(x)+M_{(9 / 8)}^{2} f(x)\right)
$$

and for all cubes $Q, R$ with $Q \subset R, x \in Q$,

$$
\left|h_{Q}-h_{R}\right| \leq C\|b\|_{* *}\left(M_{(9 / 8)}^{2} f(x)+T_{*} f(x)\right) K_{Q, R}^{2} .
$$

To obtain (2.12) for some fixed cube $Q$ and $x$ with $x \in Q$, we rewrite $[b, T] f$ :

$$
[b, T] f=\left(b-b_{Q}\right) T f-T\left(\left(b-b_{Q}\right) f_{1}\right)-T\left(\left(b-b_{Q}\right) f_{2}\right),
$$


where $f_{1}=f \chi_{(4 / 3) Q}, f_{2}=f-f_{1}$. Let us estimate the term $\left(b-b_{Q}\right) T f$ first. Take $1<r<$ $\varepsilon / \delta$. By Hölder's inequality, we have

$$
\begin{aligned}
& \left(\frac{1}{\mu((3 / 2) Q)} \int_{Q}\left|\left(b(y)-b_{Q}\right) T f(y)\right|^{\delta} d \mu(y)\right)^{1 / \delta} \\
& \quad \leq\left(\frac{1}{\mu((3 / 2) Q)} \int_{Q}\left|b(y)-b_{Q}\right|^{\delta r^{\prime}} d \mu(y)\right)^{1 / \delta r^{\prime}}\left(\frac{1}{\mu((3 / 2) Q)} \int_{Q}|T f(y)|^{\delta r} d \mu(y)\right)^{1 / \delta r} \\
& \quad \leq C\|b\|_{* *} M_{\delta r,(3 / 2)}(T f)(x) \leq C\|b\|_{* *} M_{\varepsilon,(3 / 2)}(T f)(x) .
\end{aligned}
$$

Since $T: L^{1}(\mu) \rightarrow L^{1, \infty}(\mu)$ (see [9]) and $0<\delta<1$, Kolmogorov's inequality and generalized Hölder's inequality yield

$$
\begin{aligned}
& \left(\frac{1}{\mu((3 / 2) Q)} \int_{Q}\left|T\left(\left(b-b_{Q}\right) f_{1}(y)\right)\right|^{\delta} d \mu(y)\right)^{1 / \delta} \\
& \quad \leq \frac{1}{\mu((3 / 2) Q)} \int_{(4 / 3) Q}\left|\left(b(y)-b_{Q}\right) f(y)\right| d \mu(y) \\
& \quad \leq C\left\|b-b_{Q}\right\|_{\exp L,(4 / 3) Q,(9 / 8)}\|f\|_{L \log L,(4 / 3) Q,(9 / 8)}
\end{aligned}
$$

while John-Nirenberg inequality implies that

$$
\frac{1}{\mu((3 / 2) Q)} \int_{(4 / 3) Q} \exp \left(\frac{\left|b(y)-b_{Q}\right|}{C\|b\|_{*}}\right) d \mu(y) \leq C_{0} .
$$

So there exists a positive constant $C$ such that for all cubes $Q$,

$$
\left\|b-b_{Q}\right\|_{\exp L,(4 / 3) Q,(\rho)} \leq C\|b\|_{*} .
$$

Therefore

$$
\left(\frac{1}{\mu((3 / 2) Q)} \int_{Q}\left|T\left(\left(b-b_{Q}\right) f_{1}(y)\right)\right|^{\delta} d \mu(y)\right)^{1 / \delta} \leq C\|b\|_{*} M_{L \log L,(9 / 8)} f(x) .
$$

In order to prove $(2.12)$, we only need to estimate $\left|T\left(\left(b-b_{Q}\right) f_{2}\right)-h_{Q}\right|^{\delta}$. Note that

$$
K_{Q, 2^{k}(4 / 3) Q}=1+\sum_{j=1}^{k+1} \frac{\mu\left(2^{j} Q\right)}{l\left(2^{j} Q\right)^{n}} \leq 1+(k+1) C_{0} \leq C k .
$$




\section{Commutator on nonhomogeneous space}

For $x, y \in Q$, we have

$$
\begin{aligned}
\mid(T( & \left.\left.\left(b-b_{Q}\right) f_{2}\right)\right)(x)-\left(T\left(\left(b-b_{Q}\right) f_{2}\right)\right)(y) \mid \\
\leq & C \int_{\mathbb{R}^{d} \backslash(4 / 3) Q} \frac{|y-x|^{\gamma}}{|z-x|^{n+\gamma}}\left|b(z)-b_{Q}\right||f(z)| d \mu(z) \\
\leq & C \sum_{k=1}^{\infty} \int_{2^{k}(4 / 3) Q \backslash 2^{k-1}(4 / 3) Q} \frac{l(Q)^{\gamma}}{|z-x|^{n+\gamma}}\left(\left|b(z)-b_{2^{k}(4 / 3) Q}\right|+\left|b_{Q}-b_{2^{k}(4 / 3) Q}\right|\right)|f(z)| d \mu(z) \\
\leq & C \sum_{k=1}^{\infty} 2^{-k \gamma} \frac{1}{l\left(2^{k} Q\right)^{n}} \int_{2^{k}(4 / 3) Q}\left|b(z)-b_{2^{k}(4 / 3) Q}\right||f(z)| d \mu(z) \\
& +C \sum_{k=1}^{\infty} k 2^{-k \gamma}\|b\|_{*} \frac{1}{l\left(2^{k} Q\right)^{n}} \int_{2^{k}(4 / 3) Q}|f(z)| d \mu(z) \\
\leq & C \sum_{k=1}^{\infty} 2^{-k \gamma} \frac{1}{\mu\left((9 / 8) 2^{k}(4 / 3) Q\right)} \int_{2^{k}(4 / 3) Q}\left|b(z)-b_{2^{k}(4 / 3) Q}\right||f(z)| d \mu(z) \\
& +C \sum_{k=1}^{\infty} k 2^{-k \gamma}\|b\|_{*} M_{(9 / 8)} f(x) \\
\leq & C \sum_{k=1}^{\infty} 2^{-k \gamma}\left\|b-b_{2^{k}(4 / 3) Q}\right\|_{\exp L, 2^{k}(4 / 3) Q,(9 / 8)}\|f\|_{L \log L, 2^{k}(4 / 3) Q,(9 / 8)}+C\|b\|_{*} M_{(9 / 8)} f(x) \\
\leq & C\|b\|_{*} M_{L \log L,(9 / 8)} f(x)+C\|b\|_{*} M_{(9 / 8)} f(x) .
\end{aligned}
$$

For $\rho>1$, it is easy to see $M_{(\rho)} f(x) \leq M_{L \log L,(\rho)} f(x)$. Thus

$$
\left|\left(T\left(\left(b-b_{Q}\right) f_{2}\right)\right)(x)-\left(T\left(\left(b-b_{Q}\right) f_{2}\right)\right)(y)\right| \leq C\|b\|_{*} M_{L \log L,(9 / 8)} f(x) .
$$

According to Jensen's inequality, we obtain

$$
\begin{aligned}
& \left(\frac{1}{\mu((3 / 2) Q)} \int_{Q}\left|T\left(\left(b-b_{Q}\right) f_{2}\right)(y)-m_{Q}\left(T\left(b-b_{Q}\right) f_{2}\right)\right|^{\delta} d \mu(y)\right)^{1 / \delta} \\
& \quad \leq \frac{1}{\mu((3 / 2) Q)} \int_{Q}\left|T\left(\left(b-b_{Q}\right) f_{2}\right)(y)-m_{Q}\left(T\left(b-b_{Q}\right) f_{2}\right)\right| d \mu(y) \\
& \quad \leq C\|b\|_{*} M_{L \log L,(9 / 8)} f(x) .
\end{aligned}
$$

Note that for $\rho>1, M_{(\rho)}^{2} f(x) \approx M_{L \log L,(\rho)} f(x)$. By (2.15), (2.16), and (2.23) we obtain (2.12). 
For $\left\{h_{Q}\right\}_{Q}$, we want to prove (2.13). Consider two cubes $Q \subset R$ and $x \in Q$. We denote $N=N_{Q, R}+1$. We write $h_{Q}-h_{R}$ in the following way:

$$
\begin{aligned}
\mid m_{Q}( & \left.T\left(\left(b-b_{Q}\right) f \chi_{\mathbb{R}^{d} \backslash(4 / 3) Q}\right)\right)-m_{R}\left(T\left(\left(b-b_{R}\right) f \chi_{\mathbb{R}^{d} \backslash(4 / 3) R}\right)\right) \mid \\
\leq & \left|m_{Q}\left(T\left(\left(b-b_{Q}\right) f \chi_{2 Q \backslash(4 / 3) Q}\right)\right)\right|+\left|m_{Q}\left(T\left(\left(b_{Q}-b_{R}\right) f \chi_{\mathbb{R}^{d} \backslash 2 Q}\right)\right)\right| \\
& +\left|m_{Q}\left(T\left(\left(b-b_{R}\right) f \chi_{2^{N} Q \backslash 2 Q}\right)\right)\right| \\
& +\left|m_{Q}\left(T\left(\left(b-b_{R}\right) f \chi_{\mathbb{R}^{d} \backslash 2^{N} Q}\right)\right)-m_{R}\left(T\left(\left(b-b_{R}\right) f \chi_{\mathbb{R}^{d} \backslash 2^{N} Q}\right)\right)\right| \\
& +\left|m_{R}\left(T\left(\left(b-b_{R}\right) f \chi_{2^{N} Q \backslash(4 / 3) R}\right)\right)\right| \\
= & M_{1}+M_{2}+M_{3}+M_{4}+M_{5} .
\end{aligned}
$$

Let us estimate $M_{1}$. For $y \in Q$ we have

$$
\begin{aligned}
\left|T\left(\left(b-b_{Q}\right) f \chi_{2 Q \backslash(4 / 3) Q}\right)(y)\right| & \leq \frac{C}{l(2 Q)^{n}} \int_{2 Q}\left|b-b_{Q}\right||f| d \mu \\
& \leq C\left\|b-b_{Q}\right\|_{\exp L, 2 Q,(9 / 8)}\|f\|_{L \log L, 2 Q,(9 / 8)} \\
& \leq C\|b\|_{*} M_{L \log L,(9 / 8)} f(x) \leq C\|b\|_{*} M_{(9 / 8)}^{2} f(x) .
\end{aligned}
$$

So we derive $M_{1} \leq C\|b\|_{*} M_{9 / 8)}^{2} f(x)$. Let us consider $M_{2}$. For $x, y \in Q$,

$$
\begin{aligned}
\left|T f\left(\chi_{\mathbb{R}^{d} \backslash 2 Q}\right)(y)\right| & =\left|\int_{\mathbb{R}^{d} \backslash 2 Q} f(z) k(y, z) d \mu(z)\right| \\
& \leq\left|\int_{\mathbb{R}^{d} \backslash 2 Q} f(z)(k(y, z)-k(x, z)) d \mu(z)\right|+\left|\int_{\mathbb{R}^{d} \backslash 2 Q} k(x, z) f(z) d \mu(z)\right| \\
& \leq\left|\int_{\mathbb{R}^{d} \backslash 2 Q} \frac{|y-z|^{\gamma}}{|y-z|^{n+\gamma}}\right| f(z)|d \mu(z)|+T_{*} f(x) \\
& \leq C \sup _{Q_{0} \ni x} \frac{1}{l\left(Q_{0}\right)^{n}} \int_{Q_{0}}|f| d \mu+T_{*} f(x) \leq C M_{(9 / 8)} f(x)+T_{*} f(x) .
\end{aligned}
$$

Thus

$$
M_{2}=\left|\left(b_{R}-b_{Q}\right) T f\left(\chi_{\mathbb{R}^{d} \backslash 2 Q}\right)\right| \leq C K_{Q, R}\|b\|_{*}\left(T_{*} f(x)+C M_{(9 / 8)}^{2} f(x)\right) .
$$

For the term $M_{4}$, we execute the process as in (2.21). For any $y, z \in \mathbb{R}^{d}$, we get

$$
\begin{aligned}
& \left|T\left(\left(b-b_{R}\right) f \chi_{\mathbb{R}^{d} \backslash 2 Q}\right)(y)-T\left(\left(b-b_{R}\right) f \chi_{\mathbb{R}^{d} \backslash 2 Q}\right)(z)\right| \\
& \quad \leq C\|b\|_{*} M_{L \log L,(9 / 8)} f(x) \leq C\|b\|_{*} M_{(9 / 8)}^{2} f(x) .
\end{aligned}
$$


The term $M_{5}$ can be estimated as $M_{1}$. We can obtain

$$
M_{5} \leq C\|b\|_{*} M_{(9 / 8)}^{2} f(x) .
$$

Finally we have to deal with $M_{3}$. For $y \in Q$, we have

$$
\left|b_{2^{k+1} Q}-b_{R}\right| \leq C K_{2^{k+1} Q, R}\|b\|_{*} \leq C K_{Q, R}\|b\|_{*} .
$$

Then,

$$
\begin{aligned}
\left|T\left(\left(b-b_{R}\right) f \chi_{2^{N} \backslash 2 Q}\right)(y)\right| & \leq \sum_{k=1}^{N-1} \frac{1}{l\left(2^{k} Q\right)^{n}} \int_{2^{k+1} Q \backslash 2^{k} Q}\left|b-b_{R}\right||f| d \mu \\
\leq & C \sum_{k=1}^{N-1} \frac{1}{l\left(2^{k} Q\right)^{n}} \int_{2^{k+1} Q}\left|b-b_{2^{k+1} Q}\right||f| d \mu+C \sum_{k=1}^{N-1} \\
& \times \frac{1}{l\left(2^{k} Q\right)^{n}} \int_{2^{k+1} Q}\left|b_{2^{k+1} Q}-b_{R}\right||f| d \mu \\
\leq & C \sum_{k=1}^{N-1}\left\|b-b_{2^{k+1} Q}\right\|_{\exp L, 2^{k+1} Q,(9 / 8)}\|f\|_{L \log L, 2^{k+1} Q,(9 / 8)} \\
& +C \sum_{k=1}^{N-1} K_{Q, R}\|b\|_{*} \frac{\mu\left(2^{k+1} Q\right)}{l\left(2^{k} Q\right)^{n}} \frac{1}{\mu\left(2^{k+1} Q\right)} \int_{2^{k+1} Q}|f| d \mu \\
\leq & C\|b\|_{*} M_{L \log L,(9 / 8)} f(x)+C K_{Q, R}\|b\|_{*} \sum_{k=1}^{N-1} \frac{\mu\left(2^{k+1} Q\right)}{l\left(2^{k} Q\right)^{n}} M_{(9 / 8)} f(x) \\
\leq & C\|b\|_{*} M_{L \log L,(9 / 8)} f(x)+C K_{Q, R}^{2}\|b\|_{*} M_{(9 / 8)} f(x) \\
\leq & C\|b\|_{*} M_{(9 / 8)}^{2} f(x) K_{Q, R}^{2} .
\end{aligned}
$$

Taking the mean over $Q$, we get

$$
M_{3} \leq C\|b\|_{*} M_{(9 / 8)}^{2} f(x) K_{Q, R}^{2} .
$$

By the estimates on $M_{1}, M_{2}, M_{3}, M_{4}, M_{5}$, we can get (2.13).

Let us see how from (2.12) and (2.13) one obtains (2.8). If $Q$ is a doubling cube and $x \in Q$, then we have by $(2.12)$

$$
\begin{aligned}
\left|m_{Q}\left(|[b, T] f|^{\delta}\right)-\right| h_{Q}^{\delta}||^{1 / \delta} & \leq\left(\left.\frac{1}{\mu(Q)} \int_{Q}||[b, T] f\right|^{\delta}-h_{Q}^{\delta} \mid d \mu\right)^{1 / \delta} \\
& \leq C\|b\|_{*}\left(M_{\epsilon,(3 / 2)}(T f)(x)+M_{(9 / 8)}^{2} f(x)+T_{*} f(x)\right) .
\end{aligned}
$$


Also, for any cube $Q \ni x, K_{Q, \widetilde{Q}} \leq C$, and then by (2.12) and (2.13) we get

$$
\begin{aligned}
& \left(\left.\frac{1}{\mu((3 / 2) Q)} \int_{Q}||[b, T] f\right|^{\delta}-m_{\widetilde{Q}}\left(|[b, T] f|^{\delta}\right) \mid d \mu\right)^{1 / \delta} \\
& \quad \leq\left(\left.\frac{1}{\mu((3 / 2) Q)} \int_{Q}||[b, T] f\right|^{\delta}-\left|h_{Q}\right|^{\delta} \mid d \mu\right)^{1 / \delta}+\left.|| h_{Q}\right|^{\delta}-\left.\left|h_{\widetilde{Q}}\right|^{\delta}\right|^{1 / \delta} \\
& \quad+\left.|| h_{\widetilde{Q}}\right|^{\delta}-\left.m_{\widetilde{Q}}\left(|[b, T] f|^{\delta}\right)\right|^{1 / \delta} \\
& \leq\left(\frac{1}{\mu((3 / 2) Q)} \int_{Q}\left|[b, T] f-h_{Q}\right|^{\delta} d \mu\right)^{1 / \delta}+\left|h_{Q}-h_{\widetilde{Q}}\right|+\left|h_{\widetilde{Q}}^{\delta}-m_{\widetilde{Q}}\left(|[b, T] f|^{\delta}\right)\right|^{1 / \delta} \\
& \leq C\|b\|_{*}\left(M_{\epsilon,(3 / 2)}(T f)(x)+M_{(9 / 8)}^{2} f(x)+T_{*} f(x)\right) .
\end{aligned}
$$

On the other hand, for all doubling cubes $Q \subset R$ with $x \in Q$ such that $K_{Q, R} \leq P_{0}$, where $P_{0}$ is the constant in [15, Lemma 9.3, page 143]. By (2.13) we have

$$
\left|h_{Q}-h_{R}\right| \leq C\|b\|_{*}\left(M_{(9 / 8)}^{2} f(x)+T_{*} f(x)\right) K_{Q, R} P_{0} .
$$

So by [15, Lemma 9.3, page 143$]$, we get

$$
\left|h_{Q}-h_{R}\right| \leq C\|b\|_{*}\left(M_{(9 / 8)}^{2} f(x)+T_{*} f(x)\right) K_{Q, R}
$$

for all doubling cubes $Q \subset R$ with $x \in Q$, using (2.13) again, we get

$$
\begin{aligned}
& \left|m_{Q}\left(|[b, T] f|^{\delta}\right)-m_{R}\left(|[b, T] f|^{\delta}\right)\right| \\
& \quad \leq\left|m_{Q}\left(|[b, T] f|^{\delta}\right)-h_{Q}^{\delta}\right|+\left|h_{Q}^{\delta}-h_{R}^{\delta}\right|+\left|h_{R}^{\delta}-m_{R}\left(|[b, T] f|^{\delta}\right)\right| \\
& \quad \leq C\left(\|b\|_{*}\left(M_{\epsilon,(3 / 2)}(T f)(x)+M_{(9 / 8)}^{2} f(x)+T_{*} f(x)\right) K_{Q, R}\right)^{\delta} .
\end{aligned}
$$

From the above estimates, we can obtain

$$
M_{\delta}^{\#}([b, T] f)(x) \leq C\|b\|_{*}\left(M_{\epsilon,(3 / 2)}(T f)(x)+M_{(9 / 8)}^{2} f(x)+T_{*} f(x)\right) .
$$

Now we are in the position to give the definition of weights we will consider. Here we will consider the $A_{p}(\mu)$ weights introduced by Orobitg and Pérez in [11]. So we need the assumption that $\mu(\partial Q)=0$ for any cube $Q$ with sides parallel to the coordinates axes.

Let $1<p<\infty$ and let $p^{\prime}=p /(p-1)$. We say that a weight $w$ satisfies the $A_{p}(\mu)$ condition if there exists a constant $K$ such that for all cubes $Q$

$$
\left(\frac{1}{\mu(Q)} \int_{Q} w d \mu\right)\left(\frac{1}{\mu(Q)} \int_{Q} w^{1-p^{\prime}} d \mu\right)^{p-1} \leq K
$$

And we define the $A_{\infty}(\mu)$ class as $A_{\infty}(\mu)=\bigcup_{p>1} A_{p}(\mu)$. 
Theorem 2.2. Let $0<p<\infty$, let $\rho>1, w(x) \in A_{\infty}(\mu)$ defined above, then

$$
\int_{\mathbb{R}^{d}}|T f(x)|^{p} w(x) d \mu(x) \leq C \int_{\mathbb{R}^{d}}\left(M_{(\rho)} f(x)\right)^{p} w(x) d \mu(x)
$$

holds for every function $f$ for which the left-hand side is finite.

Proof. For each $\epsilon>0$ we define the maximal operator

$$
T_{\epsilon}^{*} f(x)=\sup _{\delta>\epsilon}\left|T_{\delta} f(x)\right|
$$

We only need to prove that for $w \in A_{\infty}(\mu)$, there exist suitable constants $\alpha, \beta$, $\varepsilon$ such that

$$
w\left(\left\{x: T_{\epsilon}^{*} f(x)>(1+\beta) t, M_{(\rho)} f(x) \leq \varepsilon t\right\}\right) \leq \alpha w\left(\left\{x: T_{\epsilon}^{*} f(x)>t\right\}\right), \quad t>0,
$$

for all $\alpha^{p}<(1+\beta)^{-1}$. We may assume $f$ is nonnegative and locally integrable. Follow the idea of [11], we first consider the special case when $w=1$, then (2.42) turns to

$$
\mu\left(\left\{x: T_{\epsilon}^{*} f(x)>(1+\beta) t, M_{(\rho)} f(x) \leq \varepsilon t\right\}\right) \leq \alpha \mu\left(\left\{x: T_{\epsilon}^{*} f(x)>t\right\}\right) .
$$

Since $\Omega=\left\{x \in \mathbb{R}^{d}: T_{\epsilon}^{*} f(x)>t\right\}$ is open, we decompose it into disjoint Whitney cubes $\Omega=\bigcup_{j} Q_{j}$, where $Q_{j}$ are disjoint and $2 \rho \operatorname{diam}\left(Q_{j}\right) \leq \operatorname{dist}\left(Q_{j}, \Omega^{c}\right) \leq 8 \rho \operatorname{diam}\left(Q_{j}\right)$, and every point of $\mathbb{R}^{d}$ at most lies in $4 \rho Q_{j}$ cubes. Obviously $4 \rho Q_{j} \subset \Omega$. We will show that for given $\beta>0,0<\alpha<1$, there exists $c=c(\beta, \alpha, n)$ such that for all $j$,

$$
\mu\left(\left\{x \in Q_{j}: T_{\epsilon}^{*} f(x)>(1+\beta) t, M_{(\rho)} f(x) \leq \varepsilon t\right\}\right) \leq \alpha \mu\left(4 Q_{j}\right) .
$$

Summing over all $j$, we have

$$
\mu\left(\left\{x \in \mathbb{R}^{d}: T_{\epsilon}^{*} f(x)>(1+\beta) t, M_{(\rho)} f(x) \leq \varepsilon t\right\}\right) \leq \alpha 4^{n} \mu(\Omega) .
$$

Choose $\alpha$ such that $\alpha 4^{n}<1$, then we can obtain (2.42) in the special case. For the general case $w$, recall that if $w \in A_{\infty}(\mu)$, then by [11, Lemma 2.3, page 2017], there exist positive constants $c, \delta$ such that for all cubes $Q$ and all $E \subset Q$,

$$
\frac{w(E)}{w(Q)} \leq c\left(\frac{\mu(E)}{\mu(Q)}\right)^{\delta}
$$

Looking back at (2.44), we get

$$
w\left(\left\{x \in Q_{j}: T_{\epsilon}^{*} f(x)>(1+\beta) t, M_{(\rho)} f(x) \leq \varepsilon t\right\}\right) \leq c \alpha^{\delta} w\left(4 Q_{j}\right) .
$$

Summing again over $j$, we obtain

$$
w\left(\left\{x: T_{\epsilon}^{*} f(x)>(1+\beta) t, M_{(\rho)} f(x) \leq \varepsilon t\right\}\right) \leq c \alpha^{\delta} 4^{n} w(\Omega) .
$$

Choosing $\alpha$ such that $c \alpha^{\delta} 4^{n}<(1+\beta)^{-1}$, we can get (2.42). 
It remains to prove (2.44). Fix $j$ and let $Q=Q_{j}$ and let $r=l(Q)$. Assume that there exists $b \in Q$ such that $M_{(\rho)} f(x) \leq \varepsilon t$ (otherwise the left-hand set of (2.44) would be empty). Set $z \in \Omega^{c}$, that is, $T_{\epsilon}^{*} f(z) \leq t$ such that $\operatorname{dist}(z, Q)=\operatorname{dist}\left(Q, \Omega^{c}\right)$. By a simple computation, we get

$$
Q \subset P \equiv Q\left(b, \frac{5}{2} r\right) \subset 4 Q \subset B \equiv Q(z, 18 r)
$$

Set $f_{1}=f \chi_{B}, f_{2}=f-f_{1}$. Then for $x \in Q, \gamma>\epsilon$, by the growth condition (1.1),

$$
\begin{aligned}
\left|T_{\gamma} f_{1}(x)\right| & \leq\left|T_{\gamma}\left(f \chi_{P}\right)(x)\right|+\int_{\mathbb{R}^{d}} \frac{f \chi_{B \backslash P}}{|x-y|^{n}} d \mu(y) \leq T_{\epsilon}^{*}\left(f \chi_{P}\right)(x)+\frac{c}{r^{n}} \int_{B} f(y) d \mu(y) \\
& \leq T_{\epsilon}^{*}\left(f \chi_{P}\right)(x)+c M_{(\rho)} f(x)(b) \leq T_{\epsilon}^{*}\left(f \chi_{P}\right)(x)+c \varepsilon t,
\end{aligned}
$$

and so

$$
\left|T_{\gamma} f(x)\right| \leq\left|T_{\gamma} f_{2}(x)\right|+T_{\epsilon}^{*}\left(f \chi_{P}\right)(x)+c \varepsilon t
$$

To compare $T_{\gamma} f_{2}(x)$ with $T_{\gamma} f_{2}(z)$, we use the standard arguments. We get

$$
\begin{gathered}
\left|T_{\gamma} f_{2}(x)-T_{\gamma} f_{2}(z)\right| \leq c M_{(\rho)} f(x)(b), \\
\left|T_{\gamma} f_{2}(z)\right| \leq T_{\epsilon}^{*} f(z) \leq t .
\end{gathered}
$$

Therefore

$$
T_{\epsilon}^{*} f(x) \leq T_{\epsilon}^{*}\left(f \chi_{P}\right)(x)+(1+c \varepsilon) t .
$$

Now choose $\varepsilon$ such that $2 c \varepsilon<\beta$ and consequently

$$
\left\{x \in Q: T_{\epsilon}^{*} f(x)>(1+\beta) t, M_{(\rho)} f(x) \leq \varepsilon t\right\} \subset\left\{x \in Q: T_{\epsilon}^{*}\left(f \chi_{P}\right)(x)>\frac{\beta}{2} t\right\} .
$$

Finally, since $T_{\epsilon}^{*}$ is of weak type $(1,1)$ (see [9]), we get

$$
\begin{aligned}
\mu\left(\left\{x \in Q: T_{\epsilon}^{*}\left(f \chi_{P}\right)(x)>\frac{\beta}{2} t\right\}\right) & \leq \frac{c}{\beta t} \int_{P}|f(y)| d \mu(y) \\
& =\frac{c \mu(\rho P)}{\beta t \mu(\rho P)} \int_{P}|f(y)| d \mu(y) \\
& \leq \frac{c \mu(\rho P)}{\beta t} M_{(\rho)} f(x)(b) \\
& \leq \frac{c}{\beta} \varepsilon \mu(4 \rho Q) \leq \alpha \mu(4 \rho Q)
\end{aligned}
$$

always provided that $\varepsilon$ is chosen small enough so that $c \varepsilon / \beta \leq \alpha$. 
Lemma 2.3. Let $1<p<\infty$, let $\rho>1, w \in A_{p}(\mu)$, then

$$
\int_{\mathbb{R}^{d}}\left(M_{(\rho)} f(x)\right)^{p} w(x) d \mu(x) \leq C \int_{\mathbb{R}^{d}}|f(x)|^{p} w(x) d \mu(x) .
$$

Proof. Lemma 2.3 is a part of [5, Lemma 1]. Here we can give a more direct proof. By [6, Theorem 3], $M_{(\rho)}$ is weighted weak type $(q, q)$ if $w \in A_{q}(\mu), 1<q<\infty$. Since $w \in$ $A_{p}(\mu)$, then by [11, Corollary 2.5], there exists $\varepsilon>0$ such that $w \in A_{p-\varepsilon}(\mu)$. Finally by the Marcinkiewicz interpolation theorem, we can get the desired result.

Theorem 2.4. Let $0<p<\infty$, let $\rho>1, w \in A_{\infty}(\mu), b \in \operatorname{RBMO}(\mu)$. Then there exists constant $C$ such that

$$
\int_{\mathbb{R}^{d}}|[b, T] f|^{p} w(x) d \mu(x) \leq C \int_{\mathbb{R}^{d}}\left(M_{(\rho)} f(x)\right)^{p} w(x) d \mu(x)
$$

holds for every function $f$ for which the left-hand side is finite.

Proof. For $w \in A_{\infty}(\mu)$ and $b \in \operatorname{RBMO}(\mu)$, by the estimate for the variant of the sharp maximal function, we get

$$
\begin{aligned}
\int_{\mathbb{R}^{d}}|[b, T] f|^{p} w(x) d \mu(x) \leq & C \int_{\mathbb{R}^{d}}\left(N_{\delta}([b, T] f)(x)\right)^{p} w(x) d \mu(x) \\
\leq & C \int_{\mathbb{R}^{d}}\left(M_{\delta}^{\#}([b, T] f(x))\right)^{p} w d \mu(x) \\
\leq & C \int_{\mathbb{R}^{d}}\left|M_{\epsilon,(3 / 2)}(T f)(x)\right|^{p} w(x) d \mu(x) \\
& +C \int_{\mathbb{R}^{d}}\left(M_{(9 / 8)}^{2} f(x)\right)^{p} w(x) d \mu(x) \\
& +C \int_{\mathbb{R}^{d}}\left|T_{*} f(x)\right|^{p} w(x) d \mu(x) .
\end{aligned}
$$

Here we have to justify the second inequality, precisely

$$
\int_{\mathbb{R}^{d}}\left(N_{\delta}([b, T] f)(x)\right)^{p} w(x) d \mu(x) \leq C \int_{\mathbb{R}^{d}}\left(M_{\delta}^{\#}([b, T] f(x))\right)^{p} w d \mu(x) .
$$

This inequality can be obtained by using a good- $\lambda$ argument similar to [15, Theorem 6.2]. For brevity, we omit the details. Since $w \in A_{\infty}(\mu)$, there exists $1<r<\infty$ such that $w \in A_{r}(\mu)$. Choose $\varepsilon>0$ such that $0<\varepsilon<p / r$, then by Lemma 2.3, we have

$$
\int_{\mathbb{R}^{d}}\left(M_{\epsilon,(3 / 2)}(T f)(x)\right)^{p} w d \mu \leq C \int_{\mathbb{R}^{d}}|T f|^{p} w d \mu
$$

From Theorem 2.2 and Lemma 2.3, we can get the proof of Theorem 2.4. 
Corollary 2.5. Let $w \in A_{p}(\mu)$, let $1<p<\infty$. Then

$$
\int_{\mathbb{R}^{d}}|[b, T] f|^{p} w(x) d \mu(x) \leq C \int_{\mathbb{R}^{d}}|f(x)|^{p} w(x) d \mu(x) .
$$

Remark 2.6. Han in [5] obtained a similar result with Corollary 2.5 for higher-order commutators. But Theorems 2.1, 2.2, and 2.4 in our paper are new and are of independent interest in themselves.

\section{Acknowledgments}

The authors would like to express their deep thanks to the referee for his several valuable remarks and suggestions. This work was partly supported by NNSF of China (no. 10371080) and Scientific Research Foundation for Returned Overseas Chinese Scholars.

\section{References}

[1] W. Chen and E. Sawyer, A note on commutators of fractional integrals with $\mathrm{RBMO}(\mu)$ functions, Illinois Journal of Mathematics 46 (2002), no. 4, 1287-1298.

[2] J. García-Cuerva and J. M. Martell, Weighted inequalities and vector-valued Calderón-Zygmund operators on non-homogeneous spaces, Publicacions Matemàtiques 44 (2000), no. 2, 613-640.

[3] _ On the existence of principal values for the Cauchy integral on weighted Lebesgue spaces for non-doubling measures, The Journal of Fourier Analysis and Applications 7 (2001), no. 5, 469-487.

[4] _ , Two-weight norm inequalities for maximal operators and fractional integrals on nonhomogeneous spaces, Indiana University Mathematics Journal 50 (2001), no. 3, 1241-1280.

[5] Y. C. Han, Weighted estimates for higher-order commutators of singular integral operators on nonhomogeneous spaces, Journal of South China Normal University. Natural Science Edition 2005 (2005), no. 3, 92-99.

[6] Y. Komori, Weighted estimates for operators generated by maximal functions on nonhomogeneous spaces, Georgian Mathematical Journal 12 (2005), no. 1, 121-130.

[7] J. Mateu, P. Mattila, A. Nicolau, and J. Orobitg, BMO for nondoubling measures, Duke Mathematical Journal 102 (2000), no. 3, 533-565.

[8] F. Nazarov, S. Treil, and A. Volberg, Cauchy integral and Calderón-Zygmund operators on nonhomogeneous spaces, International Mathematics Research Notices 1997 (1997), no. 15, 703-726.

[9] __ Weak type estimates and Cotlar inequalities for Calderón-Zygmund operators on nonhomogeneous spaces, International Mathematics Research Notices 1998 (1998), no. 9, 463-487.

[10] _ Accretive system Tb-theorems on nonhomogeneous spaces, Duke Mathematical Journal 113 (2002), no. 2, 259-312.

[11] J. Orobitg and C. Pérez, $A_{p}$ weights for nondoubling measures in $\mathbb{R}^{n}$ and applications, Transactions of the American Mathematical Society 354 (2002), no. 5, 2013-2033.

[12] C. Pérez, Endpoint estimates for commutators of singular integral operators, Journal of Functional Analysis 128 (1995), no. 1, 163-185.

[13] X. Tolsa, Cotlar's inequality without the doubling condition and existence of principal values for the Cauchy integral of measures, Journal für die reine und angewandte Mathematik 502 (1998), 199-235.

[14],$L^{2}$-boundedness of the Cauchy integral operator for continuous measures, Duke Mathematical Journal 98 (1999), no. 2, 269-304.

[15] _ BMO, $H^{1}$, and Calderón-Zygmund operators for non doubling measures, Mathematische Annalen 319 (2001), no. 1, 89-149. 


\section{Commutator on nonhomogeneous space}

[16] Littlewood-Paley theory and the T(1) theorem with non-doubling measures, Advances in Mathematics 164 (2001), no. 1, 57-116.

[17] tions of the American Mathematical Society 355 (2003), no. 1, 315-348.

[18] J. Verdera, The fall of the doubling condition in Calderón-Zygmund theory, Publicacions Matemàtiques 2002 (2002), Vol. Extra, 275-292.

Wengu Chen: Institute of Applied Physics and Computational Mathematics, P.O. Box 8009, Beijing 100088, China

E-mail address: chenwg@iapcm.ac.cn

Bing Zhao: Beijing October First School, Beijing 100039, China

E-mail address: zhbi13isian@com.cn 